\title{
Membrane display and functional analysis of juxtacrine ligand-receptor signaling
}

\author{
Pradeep M. Nair ${ }^{1}$, Hai Ngu², Eric Torres ${ }^{3}$, Scot Marsters ${ }^{1}$, David A. Lawrence ${ }^{1}$, Jean-Philippe \\ Stephan $^{3, \dagger}$, Laszlo Komuves ${ }^{2}$, and Avi Ashkenazi ${ }^{1}$ \\ ${ }^{1}$ Cancer Immunology, ${ }^{2}$ Pathology, ${ }^{3}$ Biochemical and Cellular Pharmacology, Genentech, Inc., South \\ San Francisco, CA
}

${ }^{\dagger}$ Current address: Servier, France

BioTechniques 59:231-240 (October 2015) doi 10.2144/000114342

Keywords: transport; screen; juxtacrine; supported membrane display; caspase; apoptosis; death receptor

Supplementary material for this article is available at www.BioTechniques.com/article/114342.

We developed a strategy for identifying modulators of juxtacrine signaling, triggered by a cell-surface ligand displayed on synthetic lipid bilayers, via cognate receptors on apposed cells. Using readouts for receptor lateral transport and intracellular signaling, we screened a small interfering RNA (siRNA) library and identified specific receptor tyrosine kinases (RTKs) that directly or indirectly modulate apoptosis signaling by a model death ligand through its cognate death receptors. This approach may be broadly useful for studying juxtacrine cell-cell signaling systems.

Surface receptors enable individual cells to detect and respond to diverse biological cues in their surroundings, including signals emanating from diffusible factors, extracellular matrix components, or other cells. Many receptors respond in a juxtacrine manner to ligands presented on physically apposed cell membranes. This spatially controlled form of signaling imparts several biophysical characteristics, including constrained ligandreceptor geometric orientation, energetic cost to internalization of the complex, and kinetics that are heavily influenced by the proximity of the binding partners $(1,2)$. Such intercellular interactions are central to a number of biological systems and functions, including immunity (3), neuronal development (4), cell proliferation, and apoptosis (5).

Supported-membrane display strategies, in which protein ligands are presented on phospholipid bilayers deposited on underlying substrates and then engaged with receptor-expressing live cells, can faithfully recapitulate signaling by native cell-surface ligands (6-8). We recently used supportedmembrane display to study the death ligand Apo2L/TRAIL (TNFSF10), a member of the tumor necrosis factor (TNF) superfamily, which is natively expressed as a type II transmembrane protein on activated immune cells (9-11). Upon apposition with tumor cells expressing its cognate death receptors, DR4 and DR5 (12), membrane-displayed Apo2L (Apo2L.M) induced formation of microclusters of ligand-bound DR4/5, which were laterally transported at the interface between the cell and the supported membrane (Supplementary Video S1). Concomitant with this transport, the apoptosis-initiating protease, caspase-8, was recruited to receptor microclusters, leading to activation of executioner caspases-3 and -7 , and subsequent apoptotic cell death (9). Critically, Apo2L/TRAIL induced markedly more potent tumor cell death when presented on membrane surfaces as compared with its soluble form, indicating that display of this ligand on lipid membranes significantly alters the cellular response mediated by its cognate receptors. While the planar geometry of supported membranes makes them uniquely well-suited to observe microscopic clustering, this response is similar to the ligand-induced death receptor clustering at the cell surface previously associated with the pro-apoptotic receptor activity of Fas $(13,14)$. Earlier studies have shown that stimulation with Apo2L/TRAIL induces a shift of DR4 and DR5 complexes to higher molecular weight fractions (15). Our goal was to design an assay that allowed interrogation of receptor signaling in response to membranedisplayed ligands in a systematic, unbiased fashion to uncover signaling modulators of the pro-apoptotic response.

\section{METHOD SUMMARY}

Synthetic lipid bilayers displaying the membrane protein ligand Apo2L/TRAIL were used to stimulate death receptor-expressing cells in a modular, scalable format. RNA interference was used to selectively and potently silence expression of 51 receptor tyrosine kinases, and the subsequent change in response to membrane-displayed ligand was analyzed using quantitative measures of ligand-induced receptor lateral transport and intracellular signaling. 


\section{Materials and methods}

Generation of site-specifically biotinylated recombinant Apo2L/TRAIL protein

Apo2L ${ }^{\mathrm{K} 179 \mathrm{C}}$ was generated via mutagenesis of Apo2L.0 as previously described (16), produced in E. coli, and purified using a series of ion exchange and hydrophobic interaction chromatographies. The engineered free cysteine moiety was used to attach biotin site-specifically using maleimide-PEG2biotin (Life Technologies, Grand Island, NY).

\section{Generation of Apo2L.M-displaying}

\section{lipid bilayers}

Lipids dissolved in chloroform were mixed at a ratio of 99.9 mol \% 1,2-dioleoyl-sn-glycero-3-phosphocholine and 0.1 mol \% 1,2-dioleoyl-sn-glycero-3-phosphoethanolamine-N(cap biotinyl). Chloroform was then evaporated under a rotary evaporator and lipid films were dried under $\mathrm{N}_{2}$. Lipids were hydrated in phosphate-buffered saline (PBS) and extruded through $100 \mathrm{~nm}$ polycarbonate pore filters to form a monodispersed population of liposomes $100 \mathrm{~nm}$ in diameter.

Supported membranes were deposited as described previously $(9,17)$. To each well of 96 -well plates (Thermo Fisher Scientific, Pittsburgh, PA), $360 \mu \mathrm{L} 1 \mathrm{M} \mathrm{NaOH}$ was added using a VPrep Vertical Pipetting Station (Agilent Technologies, Santa Clara, CA), and plates were incubated for $1 \mathrm{~h}$. Wells were rinsed 3 times with $400 \mu \mathrm{L}$ water per rinse, using a

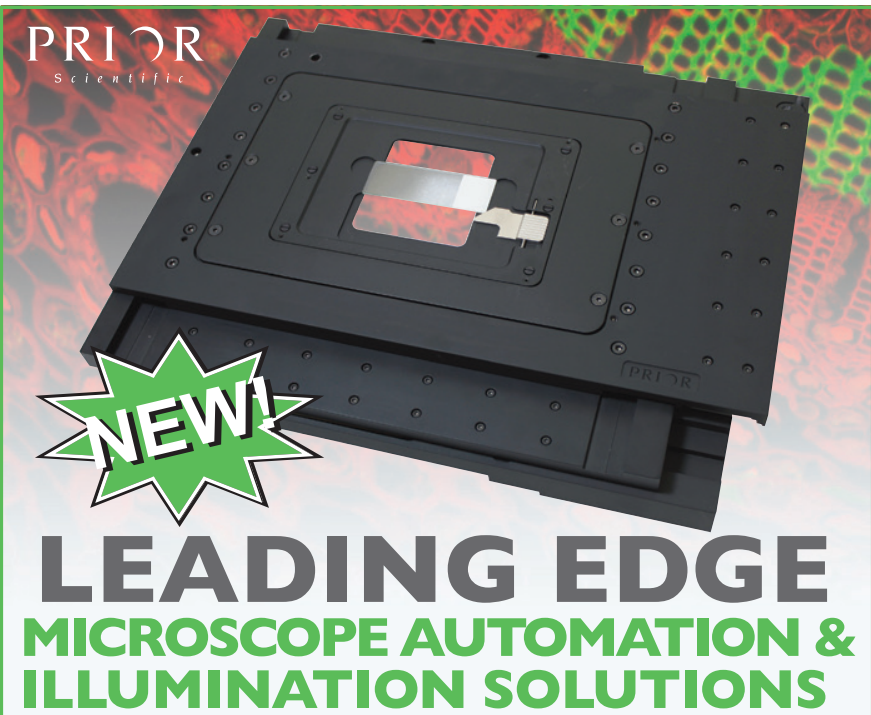

From the highest precision linear motor motorized microscope stages to the most advance fluorescence illumination systems, Prior Scientific manufactures the highest performing and most reliable microscope automation equipment available.
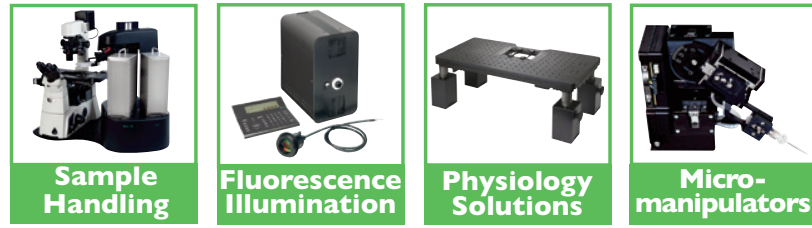

Prior Scientific, Inc. 80 Reservoir Park Dr. Rockland, MA. 02370 Tel: 800-877-2234 Web: www.prior.com
Microplate Washer (Biotek Instruments, Winooski, VT). Then, $100 \mu \mathrm{L}$ liposomes was added and incubated for $1 \mathrm{~h}$. Wells were rinsed three times with $400 \mu \mathrm{L}$ PBS per rinse, with the washer height set to ensure $100 \mu \mathrm{L}$ of solution remained in each well at all times to prevent lipid membrane exposure to air (wet rinse). This cycle was repeated with addition of 100 $\mu \mathrm{L} 0.2 \mathrm{~mol} \%$ BSA (Sigma-Aldrich, St. Louis, MO), incubation for $45 \mathrm{~min}$, wet rinse, addition of $100 \mu \mathrm{L} 2 \mu \mathrm{g} / \mathrm{mL}$ Alexa Fluor 647-conjugated streptavidin (Life Technologies), incubation for $45 \mathrm{~min}$, wet rinse, and aspiration to $200 \mu \mathrm{L}$ of solution per well for overnight storage. The following day, the well volume was aspirated to $100 \mu \mathrm{L}$; then $100 \mu \mathrm{L} 50 \mathrm{nM}$ biotinylated Apo2 $\mathrm{L}^{\mathrm{K} 179 \mathrm{C}}$ was added per well, incubated for $45 \mathrm{~min}$, and another wet rinse was performed.

Half of the plates were designated as Caspase Plates, and a wet rinse was performed 2 times with $230 \mu \mathrm{L}$ per rinse of RPMI cell culture medium supplemented with $10 \% \mathrm{v} / \mathrm{v}$ fetal bovine serum and $2 \mathrm{mM}$ L-glutamine (cell medium), supplemented with Phenol Red. The other half of the plates were designated as Transport Plates and were rinsed 2 times with $230 \mu \mathrm{L}$ per rinse of cell medium supplemented with $20 \mu \mathrm{M}$ zVAD-fmk (Tocris Bioscience, Bristol, UK).

\section{Detection of surface DR4 and DR5}

Cells were detached non-enzymatically from plates, rinsed with cold autoMACS rinsing buffer supplemented with $0.5 \%$ w/v BSA (Miltenyi Biotec, San Diego, CA), and incubated at a final concentration of $2 \mu \mathrm{g} / \mathrm{mL}$ with mouse antibodies against DR4 (4H6; Genentech, Inc., South San Francisco, CA), DR5 $(3 \mathrm{H} 3$, Genentech, Inc.), or isotype-matched controls. Free antibody was rinsed away, and cells were incubated with Alexa Fluor 647-conjugated anti-mouse IgG antibody (Life Technologies). Free antibody was again rinsed away, and flow cytometry was used to measure cell fluorescence intensities in the Alexa Fluor 647 channel.

\section{Fluorescence microscopy}

NB-7 cells were obtained from Jill Lahti (St. Jude Children's Research Hospital, Memphis, TN, USA) and retrovirally transduced to express GFP-tagged caspase-8, as described previously (18). These cells were engaged with Apo2L.M at $37^{\circ} \mathrm{C}$ and imaged using a Ti-Eclipse microscope (Nikon, Tokyo, Japan) fitted with a 100x Plan Apo oil immersion objective (1.45 NA) and a Clara CCD camera (Andor, Belfast, UK). A Cy5 filter cube was used to detect Alexa Fluor 647 fluorescence, and a GFP filter cube was used to detect GFP-caspase-8. Cells were imaged every $20 \mathrm{~min}$ for $16 \mathrm{~h}$ after addition to wells. Nikon Elements software was used for image acquisition, and ImageJ (http://imagej.nih.gov/) was used for analysis.

After small interfering RNA (siRNA) treatment, cells in Transfection Plates were incubated for 30 min with Hoechst 33342 dye (Life Technologies) and imaged at $4 \times$ magnification using an In Cell Analyzer 2000 high-content image analysis platform (GE Healthcare Bio-Sciences, Pittsburgh, PA) with a DAPI filter cube to detect Hoechst fluorescence. Nuclei were counted using In Cell Investigator software.

After Apo2L.M treatment, fixed HCT116 Bax $^{-/}$cells (obtained from Bert Vogelstein, Johns Hopkins School of Medicine, Baltimore, MD) were imaged using an ImageXpress 
A
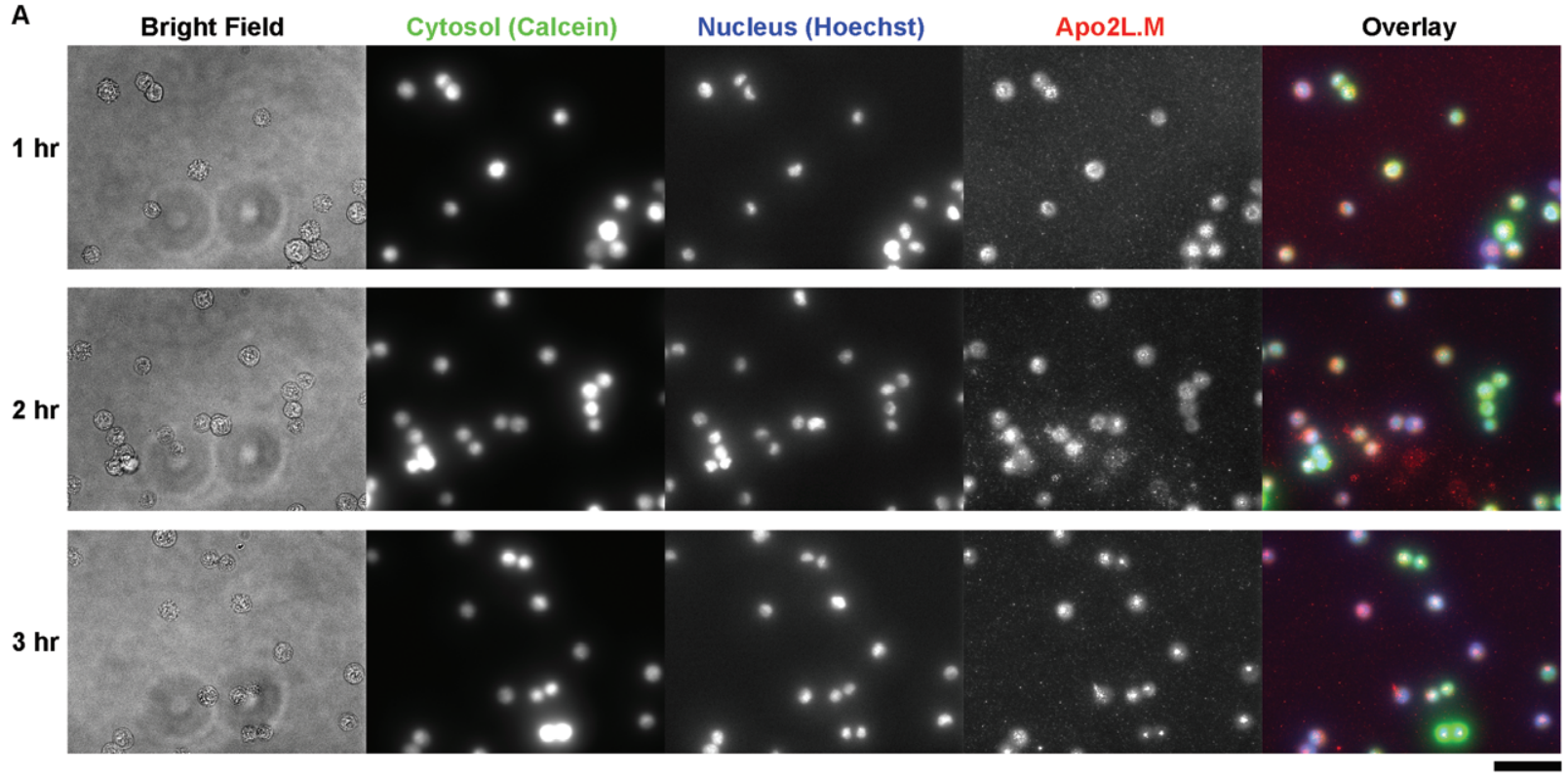

B
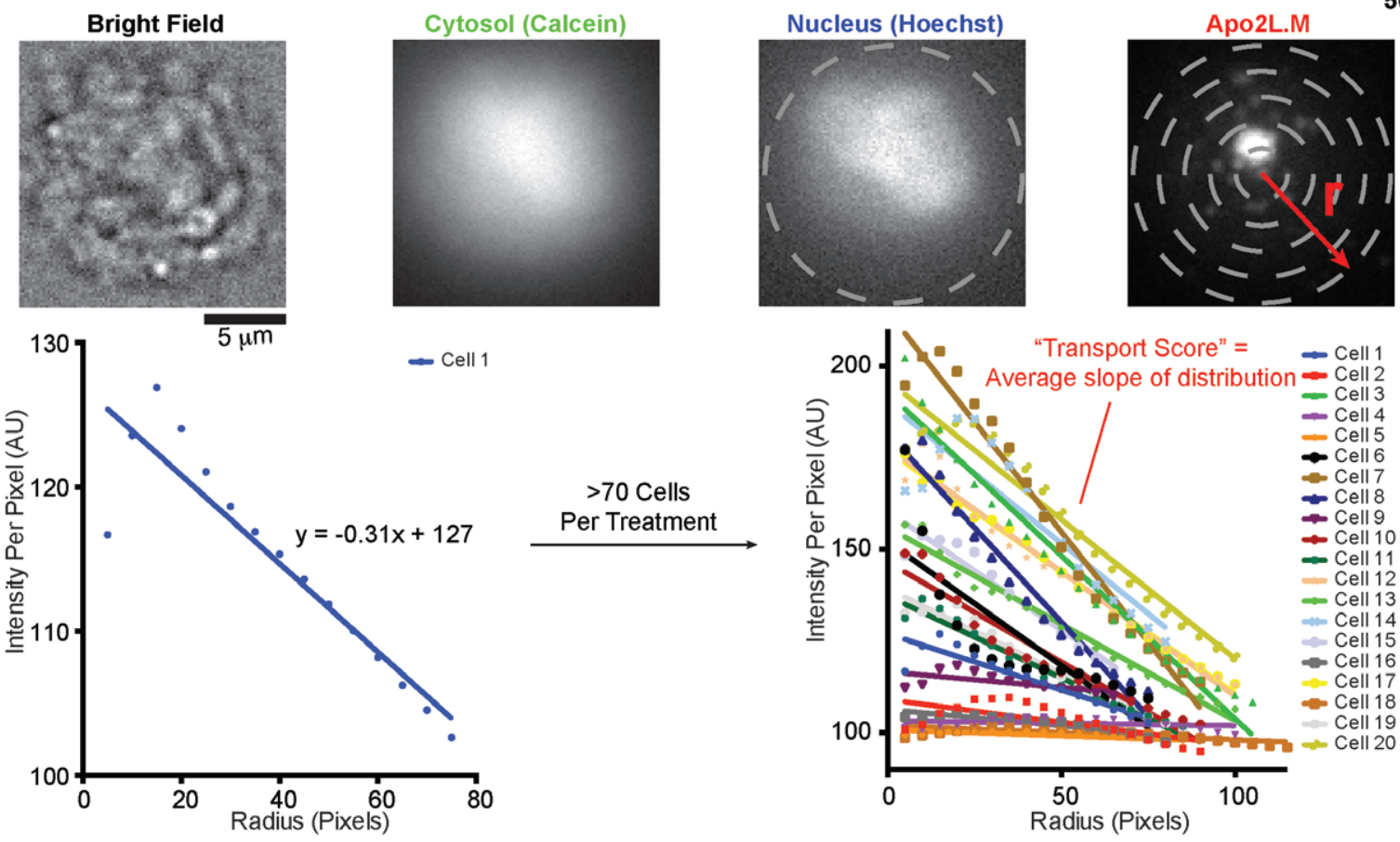

C

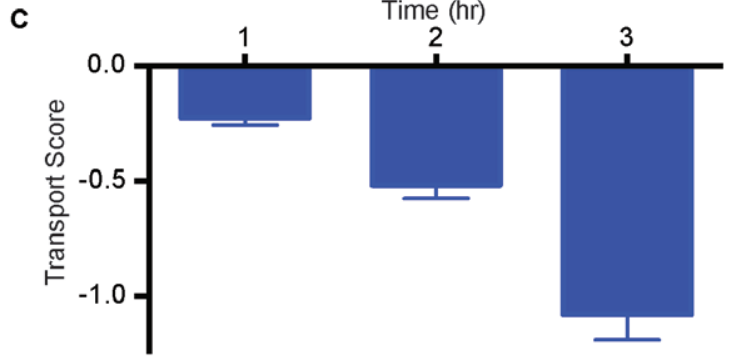
$>70$ Cells
Per Treatment

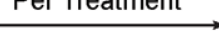

$50 \mu \mathrm{m}$

Figure 1. Quantification of Apo2L.M-induced death-receptor transport. (A) HCT116 Bax ${ }^{-1}$ cells stained with Hoechst 33342 labeling the nucleus and calcein AM labeling the cytosol, and then deposited on supported membranes displaying Apo2L.M. At the indicated time points, cells were fixed and Triton X-100 was added to remove unbound Apo2L.M. Corresponding bright field, cytosol, nucleus, and Apo2L.M channels were imaged. (B) An example image of a cell engaged with an independent Apo2L.M-displaying surface, depicting the image analysis protocol performed for each analyzed cell. The nuclear stain was used to determine cell locations within each image, and then the radial distribution of Apo2L.M underneath each cell was measured. Linear regression was performed to fit each radial distribution to a line, and the slope of this line served as a measure of death receptor transport for each cell. This process was repeated for at least 70 cells per treatment, and the average slope served as the Transport Score for the treatment. (C) Transport Scores calculated as described in (B) for cells engaged with Apo2L.M-displaying surfaces for the indicated times. Error bars indicate SEM; $n=$ at least 70 cells per time point. 
A
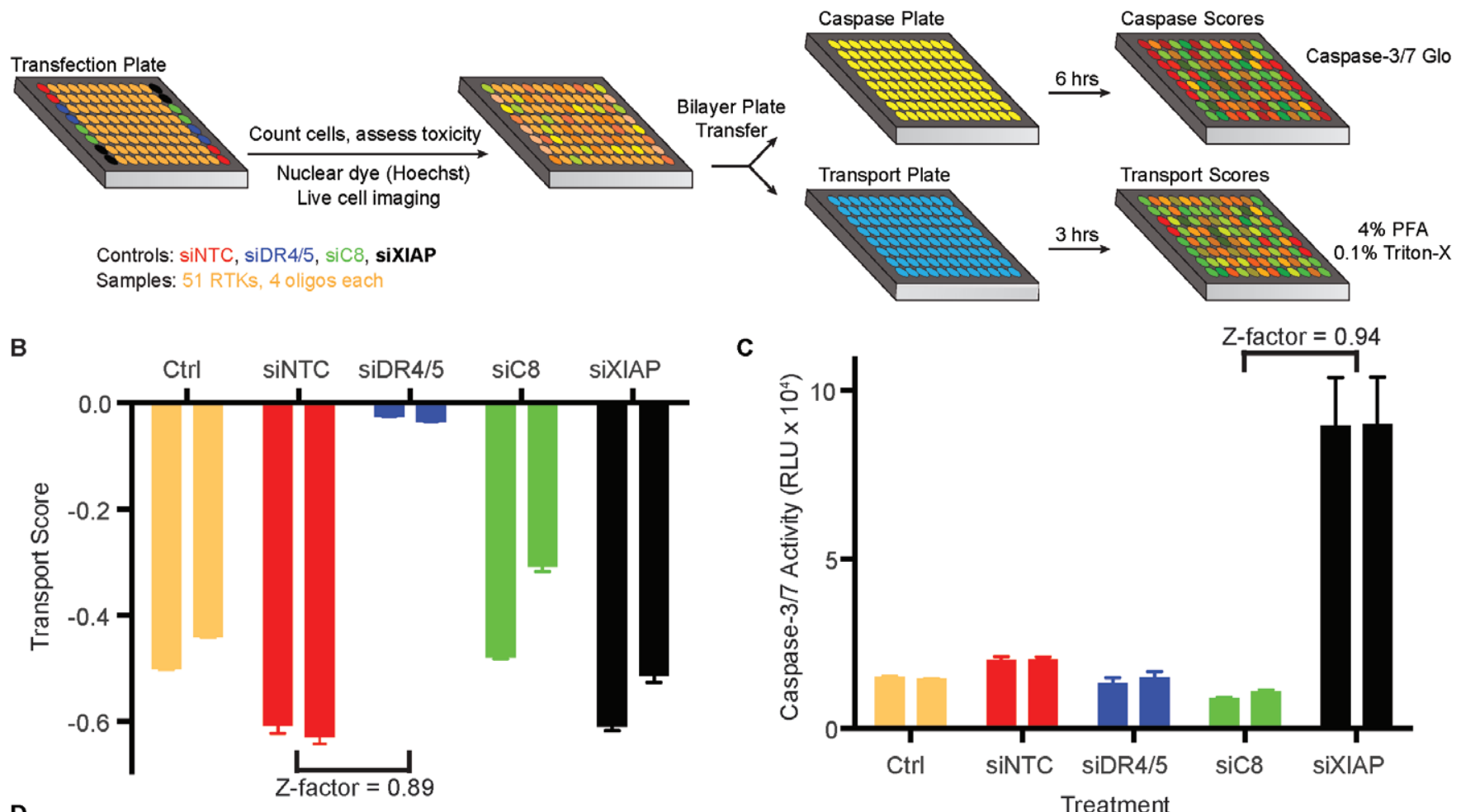

C
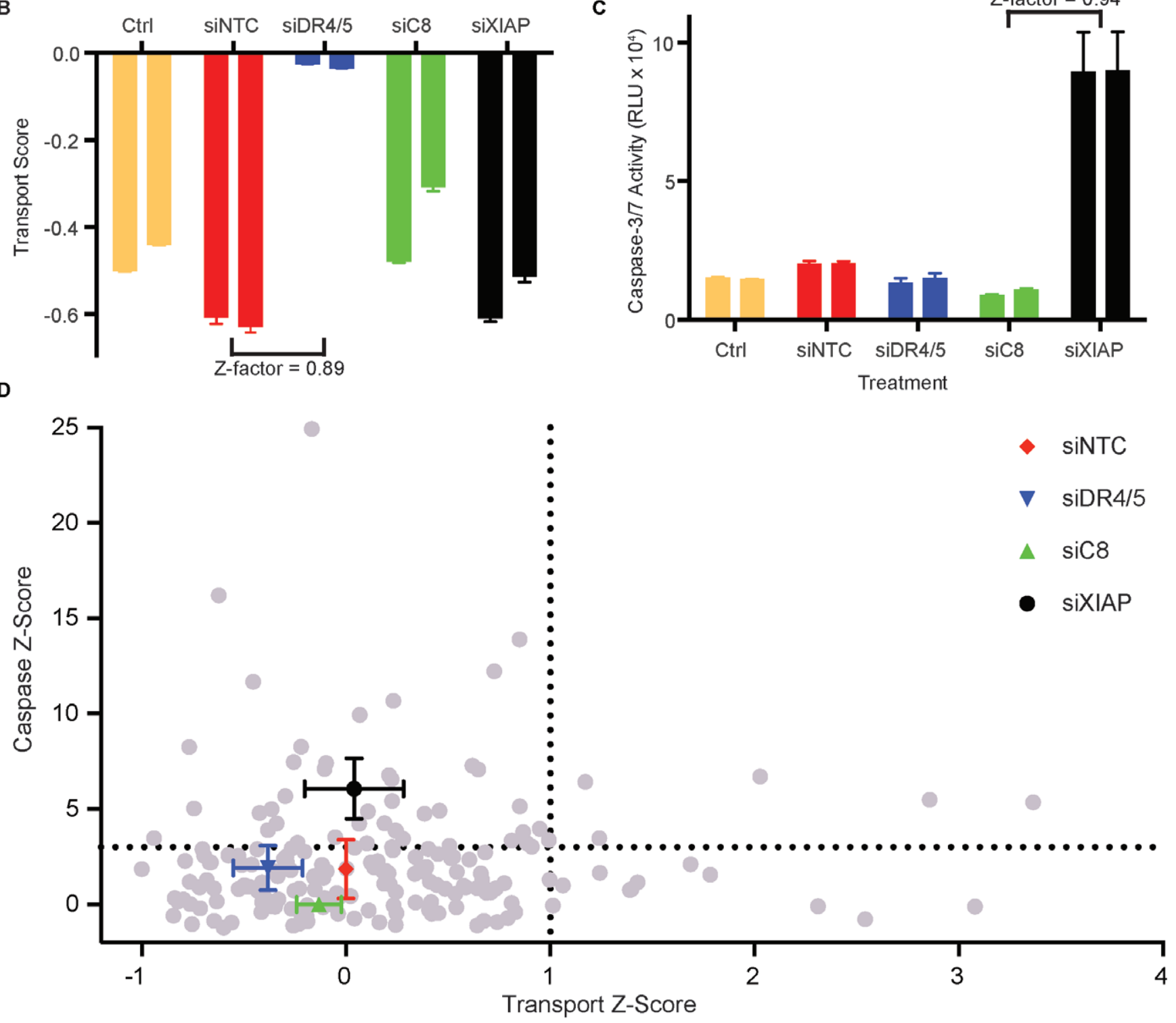

Figure 2. RNAi-treated cells are engaged with membrane-displayed Apo2L/TRAIL. (A) HCT116 Bax ${ }^{-1}$ cells were subjected to small interfering RNA (siRNA) reverse transcription of target genes for $72 \mathrm{~h}$, then stained with a cell-permeable nuclear dye, imaged to gauge siRNA cytotoxicity, detached, and added to multi-well plates in which Apo2L.M was deposited. The subsequent cell response in terms of caspase-3/7 activity and death receptor transport was measured. (B) Transport Scores for cells treated with: no treatment (Ctrl), non-targeting control (siNTC), death receptor silencing (siDR4/5), caspase-8 silencing (siC8), or XIAP silencing (siXIAP). The Z-factor for the transport readout was calculated using siNTC as the baseline control and siDR4/5 as the positive control sample. Each bar corresponds to at least 800 cells in at least 4 wells, and error bars indicate SEM for all cells. Bars indicate scores for replicate plates as a measure of plate-to-plate variability. (C) Caspase-3/7 activity for cells treated as in (B). The Z-factor for the caspase readout was calculated using siC8 as the baseline control and siXIAP as the positive control. Each bar corresponds to least 4 wells, and error bars indicate SEM for all wells. Bars indicate scores for replicate plates as a measure of plate-to-plate variability. (D) Transport and Caspase Z-Scores were calculated for 4 siRNA sequences targeting each of 51 receptor tyrosine kinases (RTKs), as well as for the siNTC, siDR4/5, siC8, and siXIAP controls. Dashed lines mark the cutoffs used in hit determination: Transport Z-score $>1$ and Caspase Z-score $>3$. 
Micro (Molecular Devices, Sunnyvale, CA) fitted with a 40x Plan Fluor ELWD air immersion objective (0.6 NA) and a Photometrics Coolsnap $\mathrm{HQ}^{2} \mathrm{CCD}$ camera. A Cy5 filter cube was used to detect Alexa Fluor 647, a FITC filter cube to detect calcein AM (Life Technologies) (in pilot experiments), and a DAPI filter cube to detect Hoechst dye. MetaXpress software was used for image acquisition.

\section{Transport assay}

After image acquisition of fixed HCT116 $\mathrm{Bax}^{-/}$cells, a custom MetaXpress analysis script was used to identify areas of each image corresponding to cells by digitally dilating the Hoechst channel to encompass the cytosol. The intensity of Apo2L.M fluorescence was measured at different radial distances from the center of each cell. The output from the MetaXpress script was processed using a custom Excel macro that used least-squares analysis to fit a line to the Apo2L.M intensity versus radial distance curve for each cell. The slope of this line served as the Transport score for each cell.

\section{Caspase activation assays}

Apo2L/TRAIL induces death-receptor oligomerization and caspase activity within minutes of stimulation $(15,18)$. Caspase- 8 activity is curtailed through TRAF2-mediated ubiquitination and subsequent proteasomal degradation (19). Thus for the initial screen, caspase-3/7 activity, a more durable response, was used as an indicator of signaling. After $6 \mathrm{~h}$ of Apo2L.M treatment, Caspase-3/7 Glo (Promega, Madison, WI) was added to cells in Caspase Plates at a 1:1 volume ratio. For the validation screen, caspase- 8 activity was also measured, using Caspase-8 Glo (Promega) added at a 1:1 volume ratio after $4 \mathrm{~h}$ of treatment-the time at which caspase- 8 activity peaks in response to Apo2L/TRAIL in this cell line (19). Both reagents were mixed at room temperature for $1 \mathrm{~h}$, and then luminescence in each well was measured using an Envision Plate Reader (PerkinElmer, Inc., Waltham, MA).

\section{SiRNA reverse transfection}

HCT116 Bax ${ }^{-}$cells were detached from tissue culture flasks using TryplE
Express, then counted and added to 96-well plates, hereafter labeled as Transfection Plates, at a density of $4 \times$ $10^{3}$ cells per well in $80 \mu \mathrm{l}$ cell medium containing phenol red. Separately, 0.1 $\mu$ L RNAiMAX (Life Technologies) was diluted to $10 \mu \mathrm{L}$ with Opti-MEM (Life Technologies), and 5 pmol of siRNA was diluted to $10 \mu \mathrm{L}$ with Opti-MEM for each well. These solutions were incubated at room temperature for $20 \mathrm{~min}$ and then combined and added to the cells in each well of the Transfection Plates (final volume $=100 \mu \mathrm{L}$ per well; final siRNA concentration $=50 \mathrm{nM}$ ) using an Oasis Liquid Handling System (Dynamic Devices, Wilmington, DE). As controls, on every Transfection Plate columns 1 and 12 contained quadruplicates of siRNAs specific for a non-targeting control (NTC); caspase-8 (C8); DR4 and DR5 (DR4/5); and X-linked inhibitor of apoptosis protein (XIAP), which inhibits caspase-3. Columns 2-11 were used for siRNA sequences targeting all human receptor tyrosine kinases (RTKs). For the primary screen, we used the On-Target Plus siRNA library (Dharmacon, Lafayette, CO) comprising 51 RTKs, each targeted by 4 individual siRNAs. For the validation screen, we added four individual siRNAs from the siGENOME library (Dharmacon), targeting each of the six RTKs identified as hits or related proteins. Cells were treated with siRNA for $72 \mathrm{~h}$ prior to Apo2L.M engagement.

Treatment with Apo2L.M

After siRNA treatment, Hoechst dye labeling and imaging to assess siRNA toxicity, cells were detached from Transfection Plates through the addition of 50 $\mu \mathrm{L}$ TryplE Express. After detachment, $150 \mu \mathrm{L}$ cell medium was added to each well. From every Transfection Plate well, $35 \mu \mathrm{L}$ medium was transferred to each of 2 duplicate Caspase Plates, and $35 \mu \mathrm{L}$ was added to each of 2 duplicate Transport Plates. Cells were then incubated for 3 or $6 \mathrm{~h}$ and assayed for Transport Scores or Caspase-3/7 Scores, respectively. For the validation screen, cells were incubated for $4 \mathrm{~h}$ and assayed for Caspase-8 Scores. Prior to Transport Score measurement, 90 $\mu$ l $16 \%$ paraformaldehyde was added per well (final concentration 4\%) and incubated for $20 \mathrm{~min}$. Then $4 \mu \mathrm{l} 10 \%$ Triton X-100 was added per well (final 
concentration $0.1 \%$ ), and plates were sealed and imaged.

\section{Data analysis}

All Transport and Caspase Scores were analyzed using ScreenSifter software (20) in conjunction with Microsoft Excel. All scores were normalized to platematched siNTCs, and then Z-scores were calculated for each siRNA sequence, either relative to the siNTC (Transport Scores), or sic8 (Caspase Scores). The entire screen was repeated $(n=2)$, and siRNA sequences were classified as hits for Transport Z-scores $>1$ or Caspase Z-scores $>3$ in at least 1 screen, with the same effect (increase or decrease in Z-score) in both screens. Genes were classified as hits if at least two siRNA sequences targeting the same gene caused a significant change in either the Transport or Caspase Scores.

\section{Results and discussion}

In order to uncover regulators of Apo2L.M-induced signaling, we designed a screening strategy based on dynamic death-receptor reorgani- zation and subsequent signaling activity in response to a laterally mobile ligand. This approach enabled the observation of altered death-receptor signaling due to targeted genetic silencing of specific RTKs, revealing regulators of this response. In contrast to fluorescent protein- or immunofluorescencebased approaches, use of a fluorescently labeled supported membranebound intermediate (e.g., streptavidin) as a surrogate marker for detecting Apo2L.M-death receptor clusters allows for the real-time observation of dynamic reorganization of endogenous receptors by live cells and has the additional advantage of requiring no rinsing steps that could detach cells from the underlying supported membranes.

Live-cell imaging of NB-7 cells expressing GFP-tagged caspase-8 revealed that upon encountering Apo2L.M at a surface concentration roughly equal to $17 \mathrm{nM}$, these cells engage the ligand via DR4 and DR5, and laterally transport Apo2L.M-DR4/5 clusters at the interface between the cell and the supported membrane

(Supplementary Video S1). This dynamic receptor reorganization event occurs in concert with caspase- 8 recruitment to death receptor complexes at the intermembrane junction, leading to caspase activation and cell death.

To uncover regulators of dynamic ligand-induced death-receptor transport, we used the HCT116 human colorectal carcinoma cell line. Unlike NB-7 cells, HCT116 cells express endogenous caspase- 8 , and wild-type HCT116 cells are sensitive to Apo2L/TRAIL-induced apoptosis in a Bax-dependent fashion (19). Thus HCT116 $\mathrm{Bax}^{-/}$cells are resistant to the pro-apoptotic ligand and wellsuited to detect sensitizers to Apo2L/ TRAIL. When these cells were engaged with Apo2L.M, ligand-induced deathreceptor transport was again observed, as evident by the increase in Apo2L.M intensity over time near the center of the interface between the cell and the supported membrane (Figure 1A).

We next developed a metric to quantify this response to membranedisplayed ligand. Cells were incubated with a cell-permeable nuclear dye, engaged with Apo2L.M-displaying

\section{Virotağ:}

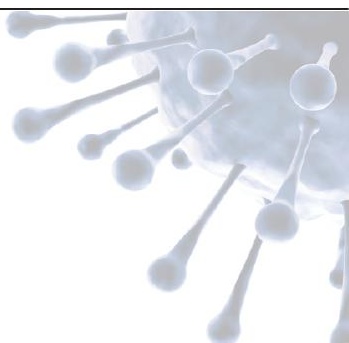

\section{Direct Antibody-Based Virus Quantification in Minutes!}

The ViroTag ${ }^{\circledR}$ detection system provides mission-critical data in real-time.

\section{Eliminates the need for time-consuming plaque titer, qPCR and other methods when combined with the ViroCyt ${ }^{\circledR}$ Virus Counter ${ }^{\circledR} 3100$.}

Immediate tracking of virus titer in your bioreactors to:

- Optimize growth conditions

- Maximize yields

- Identify problems sooner

\section{Curious? Contact us at info@virocyt.com or visit www.virocyt.com to learn more!}

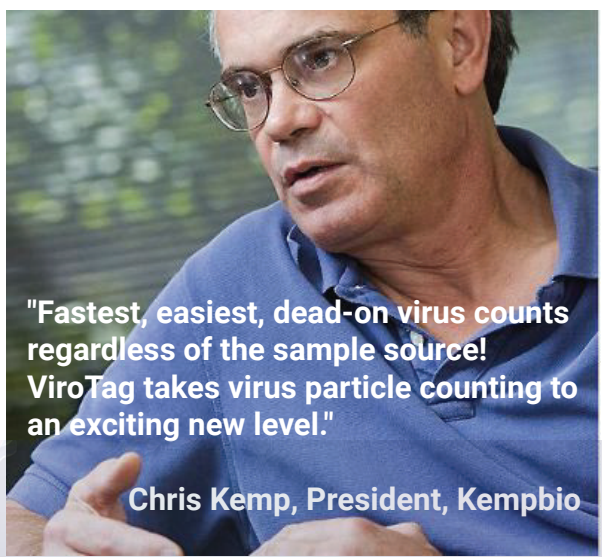


A

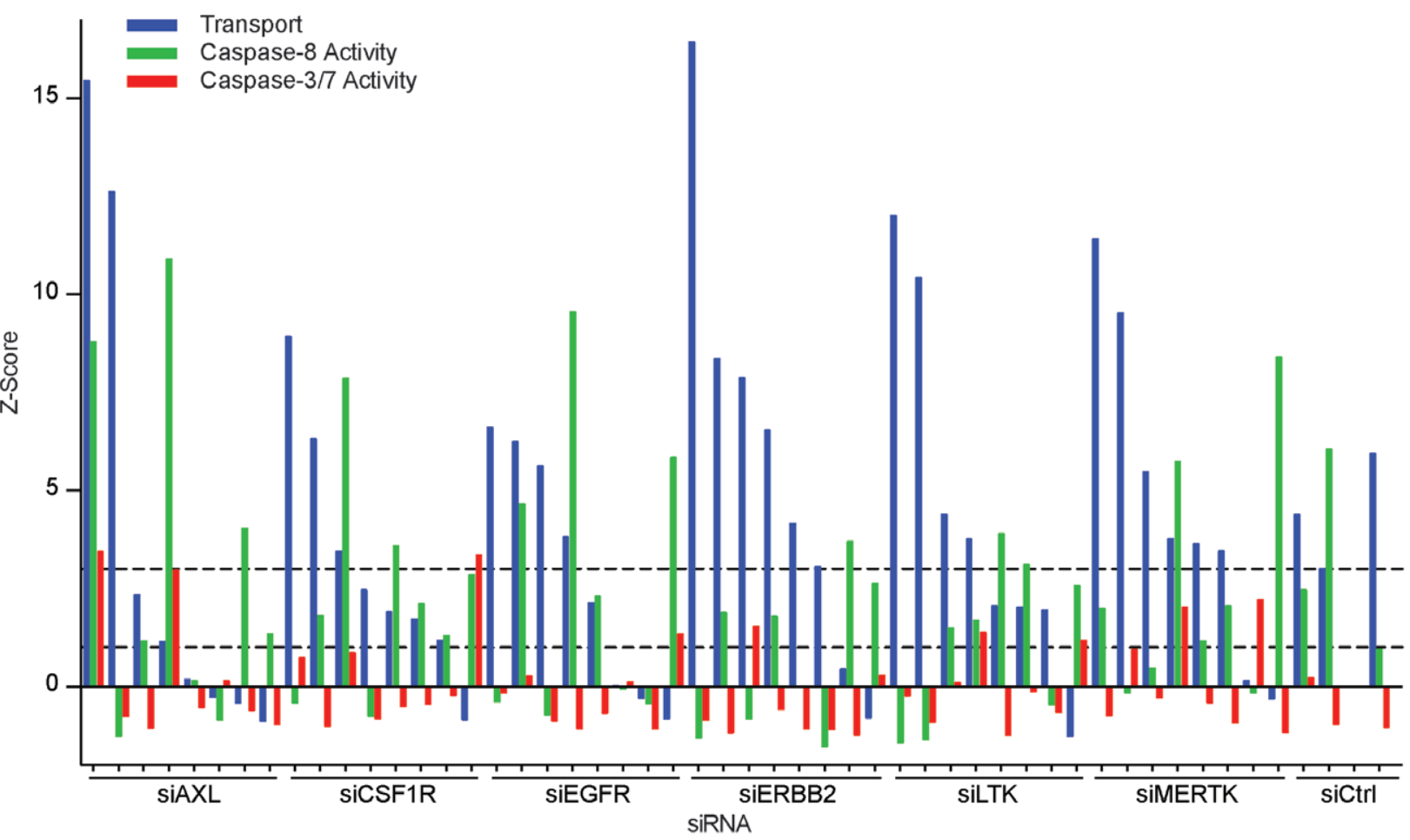

siCtrl: siC8, siDR4/5, siNTC, siXIAP

Figure 3. Validation screen confirms a role for several receptor tyrosine kinases (RTKs) in cell-extrinsic apoptosis induced by Apo2L.M. (A) The validation screen incorporated a total of eight siRNA sequences per gene for the four common hits and two related hits, and an additional caspase-8 activity readout. Dashed lines denote Z-scores of 1 (threshold for Transport Scores) and 3 (threshold for Caspase-8 and Caspase-3/7 Scores) relative to non-targeting control siRNA (siNTC).

\section{Programmable Nanoject III}

Introducing the New

\section{Easy Positive}

Micropipet Attachment No O-Rings Required

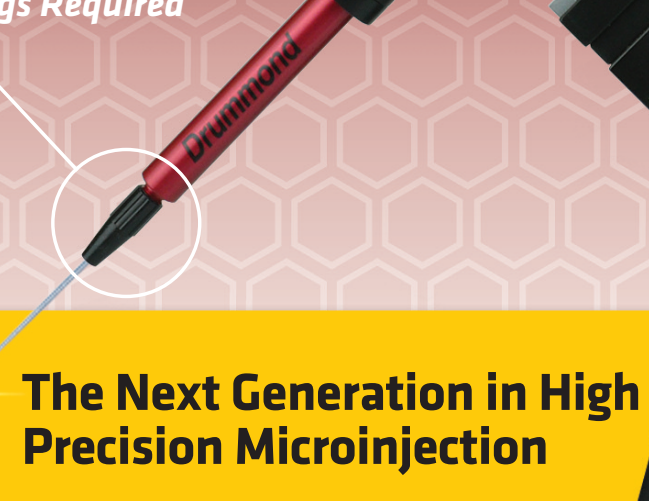

Proven Hydraulic Technology -

Ensures Consistent Injection Volumes
User Friendly Touchscreen Operation Potential Applications Expanded

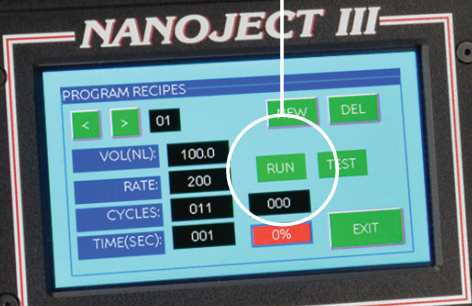

\section{IIIIII-}


surfaces, then fixed with paraformaldehyde. Triton X-100 was added to remove free ligand, and epifluorescence images were collected in the nuclear and Apo2L.M fluorescence channels. During analysis, the nuclear signal was digitally dilated to create an image mask encompassing the entire cytosol, as verified through the additional incorporation of a cytosolic dye in pilot experiments (Figure 1A). For each cell, Apo2L.M intensity was measured at different distances from the cell center (Figure $1 \mathrm{~B}$ ), and the radial distribution of Apo2L.M was plotted and fitted to a line. This process was repeated for many cells, and the average slope was labeled as the Transport Score. To validate this metric, we quantified Apo2L.M transport as a function of time and observed a progressive increase in Transport Scores (Figure 1C).

We then integrated this metric into a screening approach to investigate how juxtacrine signaling by membranedisplayed Apo2L/TRAIL might be modulated in cells expressing DR4 and DR5. Cells were first subjected to targeted gene silencing using SiRNA (21), and then engaged with Apo2L.M (Figure 2A). Based on the hypothesis that death-receptor clustering and subsequent activation may involve direct or indirect regulation by other membrane proteins, we targeted RTKs in the screen.

Expression of siRNA-targeted proteins was substantially reduced by $72 \mathrm{~h}$, as indicated by flow cytometric analysis of DR4 and DR5 in siDR4/5 cells (Supplementary Figure S1A). Although decoy receptors have been shown to bind to Apo2L/TRAIL (22) and might mediate the observed Apo2L.M transport, siRNA depletion of DR4 and DR5 abolished the observed Apo2L.M transport (Figure 2B). Additionally, while DR4 and DR5 were readily detected in HCT116 cells, decoy receptors 1 and 2 were not detectable (16). Taken together, these data confirm that DR4 and DR5 are the primary drivers of Apo2L.Minduced death-receptor transport.

Using the nuclear dye, we counted cells in each well to ensure that siRNAs did not induce significant cytotoxicity independent of Apo2L/TRAIL and to allow for exclusion of cells during analysis if cytotoxicity was observed. Cells were then detached from Transfection Plates and transferred to plates into which supported membranes displaying Apo2L.M had been deposited, labeled either Caspase Plates or Transport Plates. Transport Plates contained phenol-red-free media to reduce background fluorescence and the pan-caspase inhibitor z-VAD-fmk to prevent cell blebbing that could interfere with the readout for ligand-induced receptor transport.

One measure of the strength of a screening assay that takes into account both the dynamic range of the signal and its noise is its Z-factor (23). An ideal assay has a Z-factor of 1, and assays with Z-factors from $0.5-1$ have excellent separation between baseline and control sample readouts, relative to the noise of these data. The Z-factor for the Transport assay, using siNTC as the baseline control and siDR4/5 as the positive control sample, was 0.89 (Figure 2B), and the Z-factor for the caspase-3/7 assay, using siC8 as the baseline control and siXIAP as the positive control sample, was 0.94 (Figure 2C), suggesting these are robust readouts to identify regulators of Apo2L.M signaling.

After measuring Transport and Caspase Scores for each treatment, we analyzed the data using ScreenSifter software (20). Each Transport and Caspase Score was normalized to siNTC-treated cells. For each siRNA, normalized Transport Scores were compared with siNTC to calculate Transport Z-Scores, and normalized Caspase Scores were compared with siC8 to calculate Caspase Z-Scores (Figure 2D). These Z-Scores were then used to determine hits that significantly altered death-receptor activation in response to Apo2L.M.

Using a threshold of +3 for Caspase Z-Scores and requiring at least 2 out of 4 siRNA sequences to have the same effect, RNAi silencing of 14 RTKs increased Apo2L.M-stimulated caspase activity (Supplementary Figure S2A), sensitizing HCT116 $\mathrm{Bax}^{-/}$to Apo2L.M. Surprisingly, using a threshold of \pm 1 for Transport Z-Scores, none of the RTKs in the library decreased Apo2L.M transport. Rather, genetic silencing of two RTKs, CSF1R and MERTK, increased Apo2L.M-induced DR4 and
DR5 transport; importantly, knockdown of these proteins also increased Apo2L.M-induced caspase activity (Supplementary Figure S2, A and B). A similar, albeit weaker, association was seen in the case of LTK and ERBB2. These four RTKs, and the related proteins $A X L$ and EGFR (in the same gene families as MERTK and ERBB2, respectively) were explored further.

We next performed a validation screen using additional siRNAs against these six RTKs, with an additional caspase- 8 activity assay. The validation screen confirmed that genetic silencing of these six RTKs increased Apo2L.Mstimulated death-receptor activity (Figure 3A).

The principal advance provided by this work is a high-throughput screening approach to study juxtacrine ligand-receptor signaling. Our approach recapitulates the geometry of juxtacrine interactions by employing supported membranes deposited into multi-well plates. Demonstrating its utility, this platform enabled identification of specific modulators of pro-apoptotic juxtacrine signaling by Apo2L/TRAIL, namely several RTKs that inhibit apoptotic signaling.

This strategy is compatible with a number of well-developed technologies for selective gene silencing, including zinc-finger nucleases (ZFNs), transcription activator-like effector nucleases (TALENs), and clustered regularly interspaced short palindromic repeats (CRISPR)/Cas9-based strategies (24-26).

A growing number of juxtacrine signaling molecules are now being pursued as therapeutic targets or agents, including PD-1, PD-L1, CD40, OX40, or the cognate membraneassociated ligands of CD40 and OX40. The screening approach described here facilitates unbiased study of such ligandreceptor systems via modalities that more accurately reflect the biophysical constraints under which such signaling partners normally interact.

\section{Author contributions}

P.M.N. contributed to the conception, development, and execution of the study, data analysis, and to the writing and editing of the manuscript. H.N. 
contributed to the development and execution of the study and data analysis. E.T. contributed to the development and execution of the study. S.M. and D.A.L. contributed critical reagents and advice. J.-P.S. contributed to the development of the study and data analysis. L.K. contributed to the development of the study. A.A. contributed to the conception and development of the study, and to the writing and editing the manuscript.

\section{Acknowledgments}

We thank R. Pai for protein purification and K. Billeci, C. Chan, and Genentech's DNA and oligonucleotide synthesis labs for their support.

\section{Competing interests}

The authors declare no competing interests.

\section{References}

1. Dustin, M.L., S.K. Bromley, M.M. Davis, and C. Zhu. 2001. Identification of self through two-dimensional chemistry and synapses. Annu. Rev. Cell Dev. Biol. 17:133-157.

2. Singh, A.B. and R.C. Harris. 2005. Autocrine, paracrine and juxtacrine signaling by EGFR ligands. Cell. Signal. 17:1183-1193.

3. Monks, C.R.F., B.A. Freiberg, H. Kupfer, N. Sciaky, and A. Kupfer. 1998. Threedimensional segregation of supramolecular activation clusters in T cells. Nature 395:82-86.

4. Pasquale, E.B. 2008. Eph-ephrin bidirectional signaling in physiology and disease. Cell 133:38-52.

5. Artavanis-Tsakonas, S., M.D. Rand, and R.J. Lake. 1999. Notch signaling: Cell fate control and signal integration in development. Science 284:770-776.

6. Grakoui, A., S.K. Bromley, C. Sumen, M.M. Davis, A.S. Shaw, P.M. Allen, and M.L. Dustin. 1999. The immunological synapse: A molecular machine controlling $T$ cell activation. Science 285:221-227.

7. Narui, Y. and K. Salaita. 2013. Membrane tethered Delta activates Notch and reveals a role for spatio-mechanical regulation of the signaling pathway. Biophys. J. 105:2655-2665

8. Salaita, K., P.M. Nair, R.S. Petit, R.M. Neve, D. Das, J.W. Gray, and J.T. Groves. 2010. Restriction of receptor movement alters cellular response: Physical force sensing by EphA2. Science 327:1380-1385

9. Nair, P.M., H. Flores, A. Gogineni, S. Marsters, D.A. Lawrence, R.F. Kelley, H. Ngu, M. Sagolla, et al. 2015. Enhancing the antitumor efficacy of a cell-surface death ligand by covalent membrane display. Proc. Natl. Acad. Sci. USA 112:5679-5684.

10. Pitti, R.M., S.A. Marsters, S. Ruppert, C.J. Donahue, A. Moore, and A. Ashkenazi. 1996. Induction of apoptosis by Apo-2 Ligand, a new member of the Tumor Necrosis Factor cytokine family. J. Biol. Chem. 271:1268712690.

11. Wiley, S.R., K. Schooley, P.J. Smolak, W.S. Din, C.-P. Huang, J.K. Nicholl, G.R Sutherland, T.D. Smith, et al. 1995. Identification and characterization of a new member of the TNF family that induces apoptosis. Immunity 3:673-682.

12. Sheridan, J.P., S.A. Marsters, R.M. Pitti, A. Gurney, M. Skubatch, D. Baldwin, L. Ramakrishnan, C.L. Gray, et al. 1997. Control of TRAIL-induced apoptosis by a family of signaling and decoy receptors. Science 277:818-821

13. Siegel, R.M., J.R. Muppidi, M. Sarker, A. Lobito, M. Jen, D. Martin, S.E. Straus, and M.J. Lenardo. 2004. SPOTS: Signaling protein oligomeric transduction structures are early mediators of death receptor-induced apoptosis at the plasma membrane. J. Cell Biol. 167:735-744.

14. Kischkel, F.C., S. Hellbardt, I. Behrmann, M. Germer, M. Pawlita, P.H. Krammer, and M.E. Peter. 1995. Cytotoxicity-dependent APO-1 (Fas/CD95)-associated proteins form a death-inducing signaling complex (Dros. Inf. Serv.C) with the receptor. EMBO J. 14:55795588.

15. Wagner, K.W., E.A. Punnoose, T. Januario, D.A. Lawrence, R.M. Pitti, K. Lancaster, D. Lee, M. von Goetz, et al. 2007. Deathreceptor O-glycosylation controls tumor-cell sensitivity to the proapoptotic ligand Apo2L/ TRAIL. Nat. Med. 13:1070-1077.

16. Kelley, R.F., K. Totpal, S.H. Lindstrom, M. Methieu, K. Billeci, L. DeForge, R. Pai, S. Hymowitz, and A. Ashkenazi. 2005.

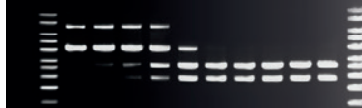

\section{Minicircle \& Plasmid DNA Service}

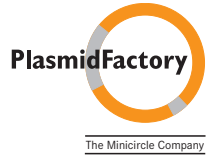

- Customized minicircle \& plasmid production

- InStock service for reporter gene plasmids and minicircles

QC including CGE service for topology analysis

(GFP, lacZ, luc, miniS/MAR etc.)

PlasmidFactory.com

PlasmidFactory GmbH \& Co. KG | Meisenstraße 96 | D-33607 Bielefeld | Germany | Fon ++49 5212997350
Receptor-selective mutants of Apoptosisinducing Ligand 2/Tumor Necrosis FactorRelated Apoptosis-Inducing Ligand reveal a greater contribution of Death Receptor (DR) 5 than DR4 to apoptosis signaling. J. Biol. Chem. 280:2205-2212.

17. Nair, P.M., K. Salaita, R.S. Petit, and J.T. Groves. 2011. Using patterned supported lipid membranes to investigate the role of receptor organization in intercellular signaling. Nat. Protoc. 6:523-539

18. Kischkel, F.C., D.A. Lawrence, A. Chuntharapai, P. Schow, K. Jin Kim, and A. Ashkenazi. 2000. Apo2L/TRAIL-dependent recruitment of endogenous FADD and Caspase- 8 to Death Receptors 4 and 5 . Immunity 12:611-620.

19. Gonzalvez, F., D. Lawrence, B. Yang, S. Yee, R. Pitti, S. Marsters, V.C. Pham, J.-P. Stephan, et al. 2012. TRAF2 sets a threshold for extrinsic apoptosis by tagging Caspase- 8 with a Ubiquitin shutoff timer. Mol. Cell 48:888899.

20. Kumar, P., G. Goh, S. Wongphayak, D. Moreau, and F. Bard. 2013. ScreenSifter: analysis and visualization of RNAi screening data. BMC Bioinformatics 14:290-301.

21. Elbashir, S.M., J. Harborth, W. Lendeckel, A. Yalcin, K. Weber, and T. Tushl. 2001. Duplexes of 21-nucleotide RNAs mediate RNA interference in cultured mammalian cells. Nature 411:494-498.

22. Ashkenazi, A. 2002. Targeting death and decoy receptors of the tumour-necrosis factor superfamily. Nat. Rev. Cancer 2:420-430.

23. Zhang, J.-H., T.D.Y. Chung, and K.R. Oldenburg. 1999. A simple statistical parameter for use in evaluation and validation of high throughput screening assays. J. Biomol. Screen. 4:67-73.

24. Gaj, T., C.A. Gersbach, and C.F. Barbas. 2013. ZFN, TALEN, and CRISPR/Cas-based methods for genome engineering. Trends Biotechnol. 31:397-405.

25. Stephan, J.P. 2014. Using RNAi screening technologies to interrogate the extrinsic apoptosis pathway. Methods Enzymol. 544:129-160.

26. Zhou, Y., S. Zhu, C. Cai, P. Yuan, C. Li, Y. Huang, and W. Wei. 2014. High-throughput screening of a CRISPR/Cas9 library for functional genomics in human cells. Nature 509:487-491.

Received 21 April 2014; accepted 29 July 2015.

Address correspondenceto Avi Ashkenazi, Cancer Immunology, Genentech, Inc., 1 DNA Way, South San Francisco, CA 94080. E-mail: aa@gene.com

To purchase reprints of this article, contact: biotechniques@fosterprinting.com 